\title{
An Indigenous Method of Securing Ventriculoperitoneal Shunt Tube in Peritoneal Cavity
}

\author{
Samir Kumar Kalra ${ }^{1, \odot ~ K r i s h n a ~ S h a h ~}{ }^{1} \quad$ Sneyhil Tyagi ${ }^{1}$ \\ 1Department of Neurosurgery, Sir Ganga Ram Hospital, New Delhi, \\ India \\ 2Department of Minimal Access Surgery, Sir Ganga Ram Hospital \\ and Ganga Ram Institute of Medical Education \& Research, \\ New Delhi, India \\ Indian J Neurosurg 2022;11:30-32
}

\author{
Suviraj John ${ }^{2, \odot ~ R a j e s h ~ A c h a r y a ~}{ }^{1}$
}

\begin{abstract}
Introduction Ventriculoperitoneal shunt (VPS) is the most common procedure used for cerebrospinal fluid (CSF) diversion in hydrocephalus. Over the years, many technical, procedural, and instrument-related advancements have taken place which have reduced the associated complication rates. Shunt block is a very common complication irrespective of the shunt system used. The abdominal end of the shunt tube gets blocked usually due to plugging of omentum onto the shunt catheter. We describe a technique of catheter fixation and placement under vision coupled with omentopexy done laparoscopically to prevent this complication.

Materials and Methods This technique was used in 23 patients ( 11 female, 12 male; range 16-73 years) afflicted with hydrocephalus from June 2016 and December 2019 after obtaining an informed consent, and the outcomes were noted in terms of shunt patency, complications, if any, and the need for revision.

Keywords

- Shunt block

- ventriculoperitoneal shunt

- laparoscopic VP shunt

Results The median operation time was 90 minutes (range 35-160 minutes). All shunt catheters were still functional after a mean follow-up of 16.5 months (range 1-34 months) and none required revision.

Conclusion Laparoscopic placement of shunt tube along with omental folding is a safe and effective technique for salvaging the abdominal end of VPS and may be helpful in reducing shunt blockage.
\end{abstract}

\section{Introduction}

Ventriculoperitoneal shunt (VPS) surgery is one of the most widely performed surgeries in neurosurgical practice, which connects the ventricles and the peritoneal cavity by a catheter. Shunt blockage is a common complication that occurs with this procedure, which could be at the level of ventricular or at abdominal level. Abdominal complications of VPS are frequent, and blockage by omentum plugging is a very common mechanism. This is due to epithelial response to the presence of catheter and omentum plugs on the shunt tube. ${ }^{1,2}$ The abdominal end of the catheter is usually inserted by a small laparotomy, placing the shunt tube in the direction of right iliac fossa. This is a blind procedure. To improve upon this technique, laparoscopic placement has been recently used where the tube placement is done under vision. It also comes very handy in revision shunt surgeries and has slowly been becoming the standard wherever feasible., ${ }^{2,3}$

A common complication which occurs with peritoneal dialysis (PD) catheters is omental wrap, which means the advancement of omentum toward and blocking the catheter. ${ }^{3}$ published online May 13, 2021
DOI https://doi.org/

10.1055/s-0041-1722826 ISSN 2277-954X.
(C) 2021. Neurological Surgeons' Society of India.

This is an open access article published by Thieme under the terms of the Creative Commons Attribution-NonDerivative-NonCommercial-License, permitting copying and reproduction so long as the original work is given appropriate credit. Contents may not be used for commercial purposes, or adapted, remixed, transformed or built upon. (https://creativecommons.org/licenses/by-nc-nd/4.0/).

Thieme Medical and Scientific Publishers Pvt. Ltd. A-12, 2nd Floor, Sector 2, Noida-201301 UP, India 
This omentum sticking to the catheter causes blockage, and to prevent this complication, omentopexy and/or omentectomy with hitching of omentum away from the peritoneal catheter have been well-described in literature. ${ }^{4,5}$

A similar technique could help prevent VPS block at abdominal end; the basic difference between the PD catheter and shunt tube is that the PD catheter is fixed and the blockage is due to omental migration and the catheter does not reach the omentum. On the other hand, VPS is a mobile tube and can migrate toward omentum and get blocked even if the omentum does not reach the shunt tube. The chances of blockage are hence technically higher than that experienced with the PD catheters. Taking a clue from this well-documented technique, if both structures are fixed and kept away from each other, this complication could be reduced.

We designed this surgical technique, whereby we did omentopexy and placed the shunt tube in an artificial tunnel of nonabsorbable sutures suprahepatically hinging to the diaphragmatic musculature, which would technically be furthest away from omentum.

\section{Materials and Methods}

This new technique was applied to 23 patients afflicted with hydrocephalus between June 2016 and December 2019. The inclusion criteria used were all patients needing VPS due to hydrocephalus, age more than 16 years, with at least a follow-up period of 6 months, and those being able to avail both surgical teams for surgery. Those excluded were patients with previous shunt blocks, those with congenital hydrocephalus, those who are less than 16 years of age, lost to follow-up, and those with infective etiology of hydrocephalus. The placement of shunt in abdominal cavity was done laparoscopically after explaining the technique and obtaining informed consent.

The indications of VPS in our study were postaneurysmal subarachnoid hemorrhage $(n=7)$, posttraumatic hydrocephalus ( $n=6)$, tumor-related hydrocephalus $(n=6)$, normal pressure hydrocephalus $(n=3)$, and postintraventricular hemorrahge hydrocephalus inplace of postintaventricular hydrocephalus $(n=1)$. Two of our patients had undergone previous abdominal surgeries as well.

The effectiveness of this technique was evaluated prospectively. The outcome parameters were perioperative complications, recurrent malfunction and catheter survival and need for revision.

\section{Technique}

All surgeries were performed under general anesthesia, and after preparation, both teams worked in tandem, with neurosurgeons operating on the cranial end and laparoscopic surgeons at the abdominal end. The shunt was tunneled and laparoscopically placed into the peritoneal cavity to suprahepatic space. An artificial tunnel was made by nonabsorbable sutures and the shunt tube was placed in it (-Fig. 1). The sutures were placed (usually 3 to 5 in number) loosely, making a sling after checking that the shunt tube was not

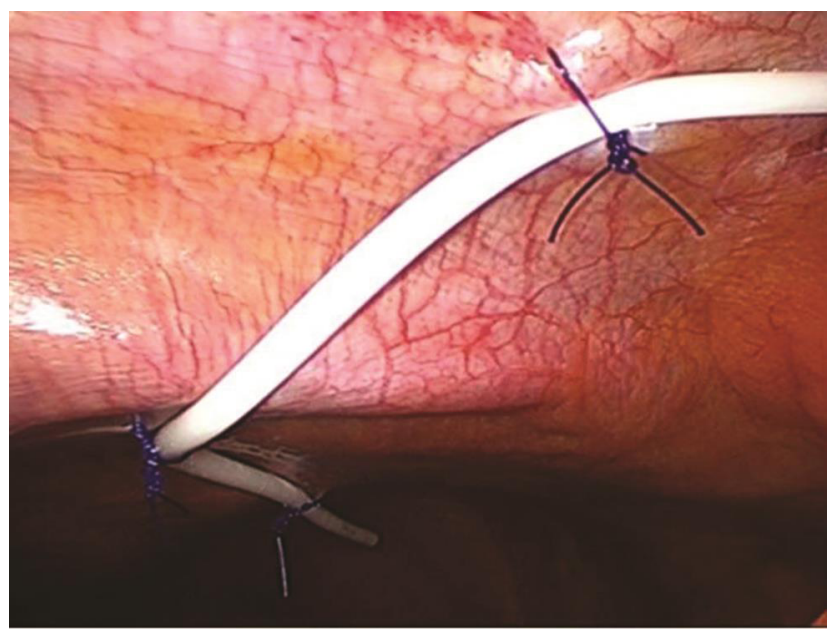

Fig. 1 Shunt tube placed in the sutured tunnel in the subhepatic space.

being tightly held by the sutures. It was also checked that the tube could be easily pulled out in the event of shunt block, if required. After placing the tube, the patency of the distal end was checked. Omentopexy (where omentum was folded onto itself) was done to prevent omental wrapping. The length of the greater omentum required for omentopexy was assessed in terms of its ability to reach the pelvis. Its distal end at the corner was then lifted and pulled in a cephalad and medial direction toward the stomach. At the end of the procedure, a sufficient distance between the omentum and catheter was ensured.

\section{Results}

The placement of shunt tube was possible in all of 23 cases. The surgical duration ranged from 35 minutes to 160 minutes (median 90 minutes). The initial duration was longer, and as the technique became more familiar the operating time, it gradually reduced. All surgeries were done by the same operating teams: neurosurgery and laparoscopic surgery. None of the cases had any bleed or anesthetic complications. Shunt catheter function was laparoscopically checked on table in all cases. The mean hospital stay was 4.0 days (3.0-11.0 days). There were no complications such as shunt infection or migration seen. After a mean follow-up of 16.5 months, none of the patients required any revision surgery.

\section{Discussion}

Despite being well-known, the mechanism of omental wrap is uncertain. It consists of tiny projections insinuating through the side holes of the catheter, obstructing its lumen and leading to catheter blockage. ${ }^{3,6}$ The mechanism of this blockage may be the same for both kind of tubes whether it is for PD catheters or VPS tube catheters. Both these are perceived as foreign bodies, leading to omental migration toward it and blockage. This incidence has been reported as between 7 to $18 \%$ in various series ${ }^{6,7}$ for such procedures. Laparoscopic placement of VPS has been used recently with good results and become a standard of care. It allows 
simultaneous inspection of peritoneal cavity and biopsy in some cases, if required; also, placement of shunt can be done under vision at the desired place. It however needs availability of another surgeon which may not always be possible. In the series by Turner et al, where they analyzed 111 patients who underwent laparoscopic peritoneal catheter placement for hydrocephalus, they concluded that the technique ensures higher shunt survival and lower infection rate. The placement of catheter is quicker, and recovery time is shorter. ${ }^{8}$ In another series by Nigim et al where the laparoscopic and open surgical approach to VPS placement was compared, it was found that the results were comparable. It was concluded that a prospective analysis is needed to assess its value as an alternate technique. It was also concluded that laparoscopic insertion is especially suited for patients who are obese and those who have undergone previous abdominal surgeries. ${ }^{9}$

A low rate of obstruction has been demonstrated by a series of primary open insertions of the catheter where prophylactic omentopexy or subtotal omenectomy had been performed. ${ }^{6,7}$ Laparoscopic omentopexy is an effective procedure for treatment of this type of omental wrap..$^{3,7}$ It is usually done with the help of a suture-passing device to skew multiple folds of the omentum or by suturing the omentum to the parietal peritoneum. Potential complications of this procedure include internal small bowel volvulus, abdominal adhesions, and hemorrhage., Omental folding is a form of omentopexy which creates a safe distance between the catheter and the omentum by shortening the latter and is associated with fewer complications. Another advantage of the folding is that the distal omentum with its slender projections is converted into a rounded edge. This lessens the risk of plugging of the catheter holes. The folded omentum does not obstruct the placement of ports or the laparoscopic views, making the resalvage a straightforward procedure if need be.

Since the relative contributions of omental and catheter movement to omental wrapping are not known, we sling the catheter in a suprahepatic tunnel and anchor it to the diaphragm. The tunnel sling allows removal of the catheter without further laparoscopy or the need to untie the subcutaneous knot. For omental fixation, we used the omental folding technique only. The complications of shunt block are also related to the etiology of hydrocephalus. The inclusion criteria used for the study also determines the incidence of block. In our study, since we have excluded the infective etiology and those who needed shunt revision, the incidence of shunt block logically is expected to be less. Moreover, the minimum aged patient is 16 years. Shunt block technically is more demanding in children and has more block-related complications and this factor also needs to be considered while analyzing our results.

\section{Conclusion}

The success of omental folding in the current study needs to be analyzed on a long-term basis. The short-term results have been satisfactory. Our study was done to test the feasibility of this new technique and quantify the role of omental plugging as a cause of the shunt tube block. The preliminary results have been encouraging. It was, however, concluded that this technique should be viewed only as an alternative to the conventional one, and its use is to be promoted when the routine technique fails.

The long-term results need to be assessed before it can be recommended as a standard technique.

The other pitfall is the required availability of a dedicated laparoscopic surgical team at all times whenever such a procedure is undertaken.

\section{Conflict of Interest}

None declared.

\section{Acknowledgment}

The authors would like to acknowledge the inputs of Dr Manu Gupta, Senior Urologist, Sir Ganga Ram Hospital, New Delhi, who also helped in conceptualizing this technique.

\section{References}

1 Acharya R, Ramachandran CS, Singh S. Laparoscopic management of abdominal complications in ventriculoperitoneal shunt surgery. J Laparoendosc Adv Surg Tech A 2001;11(3):167-170

2 Alabi A, Dholakia S, Ablorsu E. The role of laparoscopic surgery in the management of a malfunctioning peritoneal catheter. Ann R Coll Surg Engl 2014;96(8):593-596

3 Kavic SM, Segan RD, Taylor MD, Roth JS. Laparoscopic management of ventriculoperitoneal and lumboperitoneal shunt complications. JSLS 2007;11(1):14-19

4 Ogunc G. Minilaparoscopic extraperitoneal tunneling with omentopexy: a new technique for CAPD catheter placement. Perit Dial Int 2005;25(6):551-555

5 Cao W, Tu C, Jia T, et al. Prophylactic laparoscopic omentopexy: a new technique for peritoneal dialysis catheter placement. Ren Fail 2019;41(1):113-117

6 Popa F, Grigorean VT, Onose G, Popescu M, Strambu V, Sandu AM. Laparoscopic treatment of abdominal complications following ventriculoperitoneal shunt. J Med Life 2009;2(4):426-436

7 Park YS, Park IS, Park KB, Lee CH, Hwang SH, Han JW. Laparotomy versus laparoscopic placement of distal catheter in ventriculoperitoneal shunt procedure. J Korean Neurosurg Soc 2010;48(4):325-329

8 Turner RD, Rosenblatt SM, Chand B, Luciano MG. Laparoscopic peritoneal catheter placement: results of a new method in 111 patients. Neurosurgery 2007;61(3, Suppl):167-172

9 Nigim F, Thomas AJ, Papavassiliou E, et al. Ventriculoperitoneal shunting: Laparoscopically assisted versus conventional open surgical approaches. Asian J Neurosurg 2014;9(2):72-81 